\title{
Mobile operators have set ambitious targets-is it possible to boost network capacity while reducing its energy consumption?
}

\author{
Gilbert Micallef ${ }^{1 *}$, Preben Mogensen ${ }^{2}$ and Hans-Otto Scheck ${ }^{3}$
}

\begin{abstract}
While operators have to upgrade the capacity of their networks, they have committed themselves to reduce their $\mathrm{CO}_{2}$ emissions, partly by reducing their energy consumption. This article investigates the challenges faced by operators and quantifies, through a number of case studies, the impact of specific solutions and how the energy consumption trend can be expected to develop over the next decade. With different options for upgrading capacity, studies show that a hybrid macro-pico upgrade is more energy-efficient than a macro or pico only solution. The study is extended further by quantifying the possible savings by adopting an energy-efficient capacity evolution together with an equipment replacement and site upgrade strategy. Results show that network operators can get relatively close to their targets, with energy reductions of up to $40 \%$ noted. While this can be improved further through software-based energy saving features, further $\mathrm{CO}_{2}$ emissions can be offset through the use of carbon-neutral energy sources.
\end{abstract}

Keywords: Green radio, energy saving, network evolution, network upgrade, HSPA, LTE, base station site, remote radio head

\section{Introduction}

With the increasing importance of wireless communications, the need for green radio has gained considerable traction. The reduction of $\mathrm{CO}_{2}$ emissions has become a global objective, with governments, companies, and the general public, all expected to play a role and adhere to the guidelines set by the Kyoto protocol. Within many industries, emissions are directly associated with the energy consumption. To some extent, the growth of an industry can be measured through increasing $\mathrm{CO}_{2}$ emissions, which for the ICT industry is expected to double its every 4-6 years.

The telecommunications industry has taken a bold position in reducing its $\mathrm{CO}_{2}$ emissions, primarily by reducing the energy consumption. Energy costs have soared, making energy bills a burden for mobile network operators (MNOs). Besides, with the expected growth in traffic, MNOs have also got to invest and upgrade their

\footnotetext{
* Correspondence: gmi@es.aau.dk

'Radio Access Technology, Department of Electronic Systems, Aalborg

University, Niels Jernes Vej 12 A6, 9220 Aalborg $\varnothing$, Denmark

Full list of author information is available at the end of the article
}

networks, which inherently further increases the energy consumption. Besides the financial gains, a commitment in reducing the energy consumption also plays a public relations (PR) role. Many of the major MNOs have pages dedicated to 'Corporate Social Responsibility' in which they express commitment [1] towards the climate, environment, and a variety of other work ethics. In regard to reducing their carbon footprint, MNOs go a step further by setting specific targets and timelines, with Telenor [2] and Vodafone [3] aiming to reduce their carbon footprint by 40 and $50 \%$, respectively.

For these reasons, MNOs and equipment vendors alike have been investigating methods for reducing the energy consumption of mobile networks, kick-starting the concept of green radio. The need for reducing the energy consumption provides an opportunity for equipment vendors, who compete in offering diverse, reliable, feature-rich, and energy-efficient equipment for supporting the deployment of new networks and the upgrade of existing ones.

\section{Springer}

(C) 2012 Micallef et al; licensee Springer. This is an Open Access article distributed under the terms of the Creative Commons Attribution License (http://creativecommons.org/licenses/by/2.0), which permits unrestricted use, distribution, and reproduction in any medium, provided the original work is properly cited. 


\section{Base station site overview}

In mobile networks, more than $80 \%$ of the energy is consumed by the network infrastructure [2], of which more than $70 \%$ at the base station sites $[4,5]$. These sites are on the access part of the network and host equipment that enables wireless transmission and reception, connecting subscriber mobile terminals to the core network. The reason why these sites are responsible for so much of the overall energy consumption is twofold. Besides having to deploy a large quantity of such sites, to ensure full network coverage, these sites are very energy inefficient. Assuming that the effective transmitted power of a typical 3-sector base station site is in the order of $120 \mathrm{~W}$, while the total input power for the site is in excess of $2 \mathrm{~kW}$, this gives an efficiency of just $6 \%$. Thus, the biggest saving opportunities are likely to be achieved from optimizing base stations.

Besides the modules providing the core communication functions, base station sites also host components that ensure the equipment is kept safe, in adequate ambient conditions, and protected from any external interruptions. While all these components are required to ensure reliable communication networks, they all add energy overheads, increasing consumption and reducing the efficiency of each site. Major technological improvements have allowed for the availability of more energyefficient equipment. For instance, within base station sites, active cooling is considered to consume around $30 \%$ of the energy. Improvements have allowed for equipment to support higher operational temperatures, reducing or in some cases eliminating the need for active cooling. Other energy-related improvements within base station sites include more efficient rectifiers, battery backup units, and system designs. Overall, advancements in technology have allowed for more compact, flexible, and efficient equipment packed with a wider array of features.

As shown in Figure 1, equipment in a base station site can be represented through a modular structure. With regard to the core communications equipment, this is composed of two modules, the RF module and the systems module $(\mathrm{SM})$. The latter provides all functionalities related to baseband processing, control, and backhaul transmission to the core network. The RF module houses the power amplifiers, which in a $3 \mathrm{G}$ base station are responsible for $50-65 \%$ of the energy consumption. This is partly due to the need of power amplifiers to compromise efficiency for linearity [6]. Different site configurations exist, depending on particular operator requirements, and restrictions at site locations. A further energy loss at these sites is attributed to long feeder cables connecting the RF module to the antenna. This is due to dielectric losses and skin-effect, which is mainly dependent on the transmission frequency and cable length [7]. In most sites, feeder cable losses of about $50 \%(3 \mathrm{~dB})$ are assumed [8], requiring the RF module to transmit at higher power (double) in order to ensure the desired power at the antenna. A solution to this is to install the RF module in close proximity to the antenna, with an optical connection linking it back to the system module. As illustrated in Figure 2, these are known as remote radio head (RRH) units, and are often noted as small boxes mounted on the antenna mast. Due to a number of practical restrictions, the use of RRH units is not possible at all sites, for reasons that may include space and positioning on the mast, rental agreements, and visual pollution. It should be noted that even with RRH units, some losses still arise from the shorter jumper cables, and connectors.

As in [4], a linear base station site power model is used to estimate the consumption of the network. The model is based on measurements carried out by an equipment vendor and considers only the RF module, and SM. The model can be split into two main components, a load dependent and independent component. Measurements show that the load-independent component is the most dominant, meaning that even at very low load the power consumption of a site remains relatively high. The comparison of power consumption at different loads is graphically represented in Figure 3. The load-independent component arises from the base power that is required to run the equipment itself, prior to any communications. Based on the transmitted power required at the antenna, the PA has to be biased in a way to overcome any feeder cable losses.

$$
\begin{aligned}
& P_{\text {BTS }}(\text { Watts })=N_{\text {Sectors }} *\left[P_{\text {Load_Indep }}+\left(\text { Load } * P_{\text {Load_Dep }}\right)\right] \\
& \left(P_{\text {load_Indep }}\right)>\left(P_{\text {Load_Dep }} \propto P_{\text {PA_Out }}\right) \\
& P_{\text {Antenna }}=P_{\text {PA_Out }}-P_{\text {Feeder_Loss }}
\end{aligned}
$$

With base station sites identified as main energy consumers, a number of options for improving their efficiency include: hardware improvement, site design, software features, and deployment optimization.

\subsection{Base station switch off for energy saving}

With traffic patterns noted to vary over a period of 24$\mathrm{h}$, the relation of power consumption with load suggests that an efficient way to save energy during hours of low traffic is to switch off a number of the base station sites [9]. While in residential areas, the period of low traffic can be expected during night time, this can change depending on the area. For instance an industrial area is 


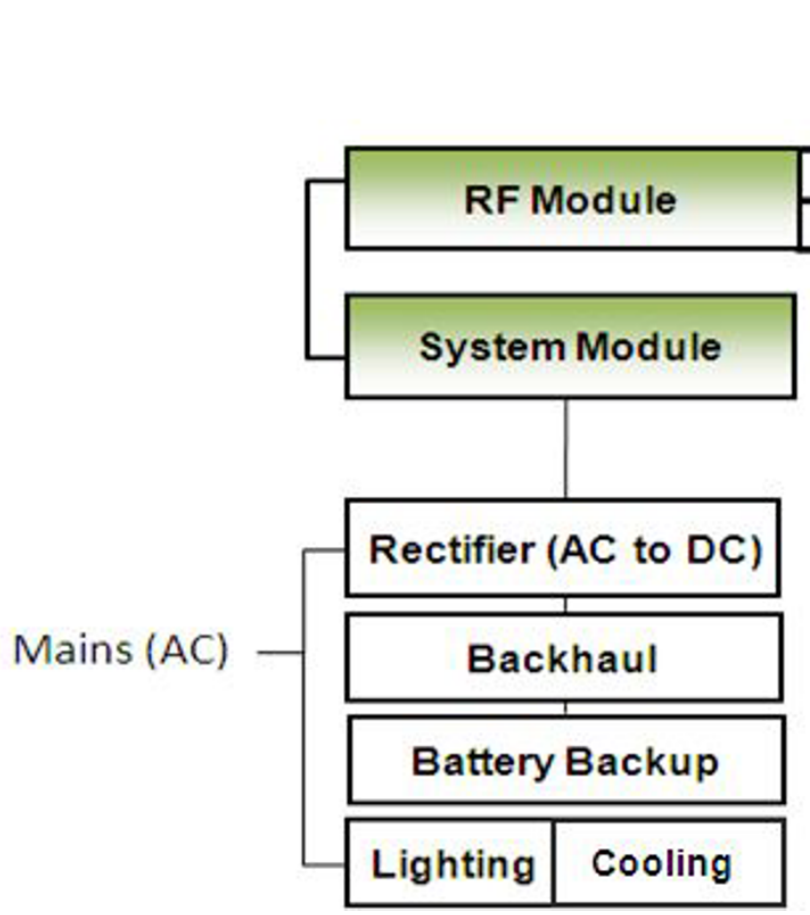

Figure 1 Modular overview of the components in a typical macro base station site. The RF and system modules are the components required for a base station site to carry out its core communication functionalities.

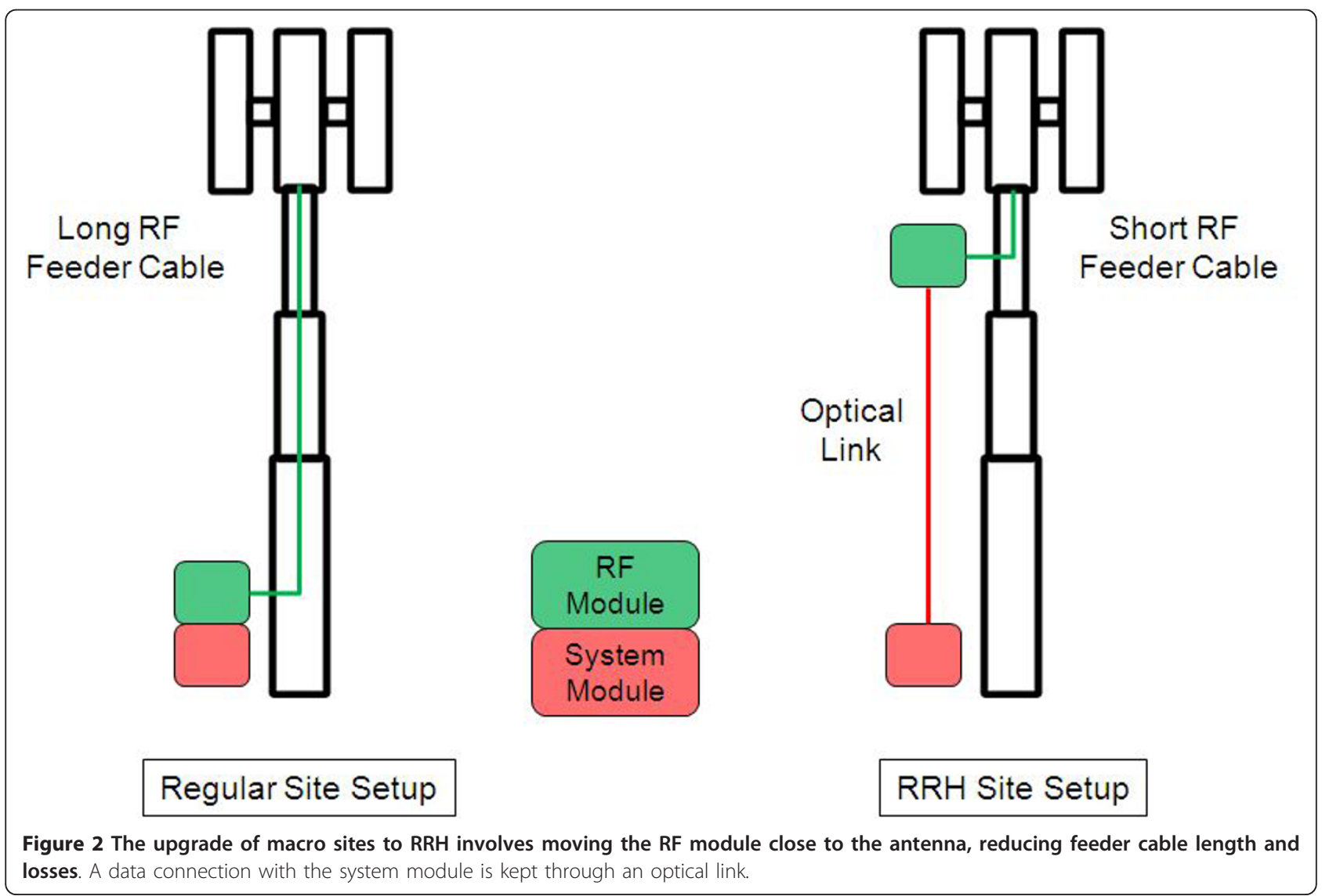




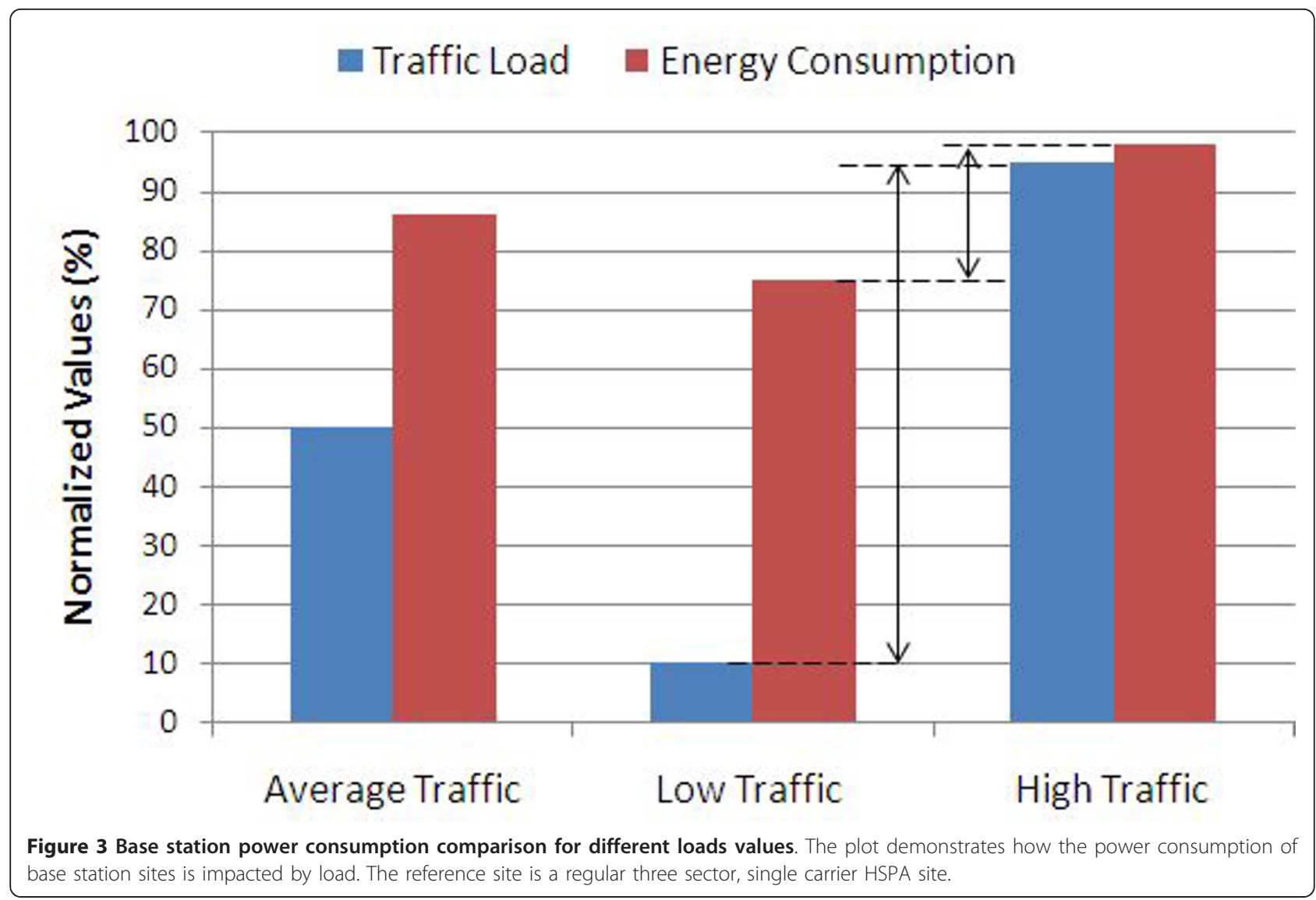

expected to have less traffic already in the late afternoon period and on weekends when people are away from work. Such a feature would, based on the conditions of the network, select a number of sites that have a load less than a pre-defined threshold, and systematically deactivate core components at the site.

In a dedicated study [10], results show that over a 24$\mathrm{h}$ period, switching off sites, or individual sectors, can result in energy savings of around $30 \%$. The idea is to power off components that consume most energy, in particular the RF power amplifiers, and leave active only equipment required for triggering a wakeup mechanism. The amount of savings possible is dependent on the area, traffic patterns, and network topology. A dense urban area with high site density is the most suitable scenario for such a feature, specifically when applying the feature to capacity enhancing sites. While this can be regarded as one of the most effective methods for reducing the energy consumption, this may have an impact on the performance of the remaining sites. It is undesired for a network operator to have subscribers note a difference in services, especially during hours when users would expect better network conditions. Simulation results show that even though the network can still guarantee a minimum required data rate, the energy savings through site switch off comes at the cost of a $25 \%$ reduction in average user data rate. Such an impact on network performance can be limited by enabling the feature in very dense urban areas (intersite distance approximately $300 \mathrm{~m}$ ), and limited to pure capacity enhancing sites. A further issue with such a feature is that existing equipment is limited in how fast it can be switched, with existing delays in the order of a few seconds. Other issues also being investigated include methods for transferring control between adjacent sites and the procedure of going into and out of sleep mode.

\section{Network capacity evolution}

The upgrade of UMTS networks to HSPA allows for MNOs to provide reliable high speed data services, dubbed 'mobile broadband'. A variety of smartphones (particularly the iPhone) provided an enriched user experience, and together with flat-rate pricing for mobile broadband, were among the reasons why mobile broadband took off when it did. Since then, mobile operators have been reporting annual traffic growths over their networks ranging from 300 to $700 \%$ [11]. Besides an increase in the number of broadband users, each user is consuming more traffic, which can be attributed to an increase in the number of available 
devices (e.g., eBook readers, laptops, GPS systems, cars), and the amount of dedicated content, especially multimedia (e.g., YouTube) and social networking (e.g., Facebook), being made available.

As a result of this sustained data traffic growth, some MNOs are finding themselves in a situation of approaching, in some areas, their network capacity limit. In an attempt to avoid or at least delay this from happening, some MNOs have started to limit or abolish completely their unlimited data plans [12]. At the same time network upgrades of various types are being carried out where necessary. In countries where licenses have already been auctioned off, operators have also started rolling out and testing LTE. By utilizing a more flexible frequency multiplexing technique (OFDM) and advanced antenna techniques, LTE can use different carrier bandwidths of up to $20 \mathrm{MHz}$, resulting in reduced latency, and a boost in data rates beyond the $100 \mathrm{Mbps}$ mark [13]. Since the uptake of any new technology could take a number of years, until the penetration rate of LTE compatible devices reaches certain levels [14], traffic can still be expected to grow on the HSPA layer. As the number of network layers increases, MNOs have to manage and maintain all layers (including GSM) prioritizing different resources and service levels to a variety of subscriber groups.

\subsection{Available capacity options}

A network site with limited capacity can result in subscribers experiencing low data rates, long delays, and in some cases no connection. In order to avoid this, MNOs plan ahead, estimating traffic growth, and upgrade networks. Network capacity is increased by upgrading existing sites, and/or through the deployment of additional sites. When possible, upgrading existing sites is preferred, since already owning the site makes it logistically and financially simpler than commissioning an entirely new site.

Assuming an existing HSDPA network and the availability of additional spectrum, MNOs can boost network capacity by increasing the number of active carriers ( 5 $\mathrm{MHz}$ ). Within some equipment versions, these can be supported within the same unit. Alternatively, if additional spectrum is not available, existing sites can be upgraded through sectorization, typically going from a 3 to 6 sector site. From a capacity point of view, doubling the available spectrum effectively doubles the capacity of the site, whereas increasing the number of sectors improves the spectral efficiency, but results in lower capacity gains. Another option for operators is to deploy additional sites, which can vary in type, depending on the expected and type of traffic requirements in the area. While traditional macro sites can cover large areas, smaller micro or pico sites are intended for dense urban areas to provide high capacity hotspots. Small site deployment, such as outdoor pico sites, reduces the extent to which surrounding macro sites need to be upgraded. When such upgrades are not enough, MNOs can rollout a new network technology (LTE), which is likely to be added at existing sites.

MNOs are faced with the need to make decisions about where, how, and when to upgrade networks, in an attempt to define an appropriate evolution path. As in every other business, operators balance network investment decisions around performance and costs. However, since operators have committed themselves to reduce energy consumption, this new third dimension has to now also be considered when making these decisions.

\subsection{An energy-efficient approach to network evolution}

To establish which network evolution path is the most energy-efficient, investigations are carried out through case studies based on dense urban European networks. Different techniques are considered and compared through detailed system-level downlink network simulations. Independent on the evolution path, simulations are aimed at having the network provide the same performance. This is achieved by assigning a key performance indicator (KPI) which is used throughout all cases. This KPI is referred to as 'user satisfaction rate', which gives the percentage of active users within the network area that can achieve a pre-defined minimum data rate. For the network to be considered as having a satisfactory performance, at least a $95 \%$ user satisfaction rate is required. This allows for a fairer energy comparison for the different evolution paths. A full buffer traffic model is used, and all base station sites are assumed to be running at $100 \%$ load. With regard to the radio resource management (RRM) algorithm, its first cycle attempts to maximize the percentage of satisfied users. Since full load is assumed, requiring all resources to be utilized, if all users achieve this minimum data rate, remaining resources are shared in a round robin fashion. While different topologies can achieve the target KPI, running sites at full load allows distinction with regards to average network data rate and hence an energy-efficiency measure. In addition, this also provides a worst case scenario from an interference point of view. Busy hour traffic is simulated by adding a number of active users within the network area. Through statistical analysis of actual traffic data, a traffic density map of the area is generated, and then used to distribute active users accordingly. A more comprehensive view of the main parameters is presented in Table 1.

In the first network evolution scenario investigated [15], macro sites are upgraded by increasing the number of carriers, and finally MIMO. In these case studies, MIMO performance is modeled through a second SINR 
Table 1 Overview of the network layout, and parameters used in setting up the system level Monte Carlo based network simulator

\begin{tabular}{ll}
\hline Cellular layout & Realistic dense-urban scenario with $\mathbf{3 5}$ sites in the investigated area. Majority of sites have $\mathbf{3}$ sectors \\
\hline Simulation pixel restitution & $25 \times 25 \mathrm{~m}^{2}$ \\
Macro path loss model & Cost-Hata model $+20 \mathrm{~dB}$ penetration loss for indoor users \\
Pico path loss model & $3 \mathrm{GPP}$ outdoor-to-indoor model for all users \\
Traffic model & Full Buffer, with minimum data rate requirement of $512 \mathrm{kbps}$ \\
Simulation KPI & $95 \%$ user satisfaction (or $5 \%$ network outage) \\
Transmission power macro/pico & $43 \mathrm{dBm} / 30 \mathrm{dBm}$ per carrier (transmission at antenna) \\
Macro antenna (Gain) & $3 \mathrm{D}$ antenna pattern with tilting values from actual network (14 dBi) \\
Pico antenna (Gain) & Omni-directional antenna (5 dBi) \\
Single carrier & $2150 \mathrm{MHz}, 5 \mathrm{MHz}$ bandwidth \\
UE receiver type & $1 \times 2 \mathrm{Rx}$ diversity with $2 \mathrm{~dB}$ body loss and $1 \mathrm{~dB}$ implementation margin \\
Multi-user scheduling gain & $1 \mathrm{~dB}$ \\
Feeder cable loss (with RRH) & $3 \mathrm{~dB}(1 \mathrm{~dB})$ \\
\hline
\end{tabular}

to throughput mapping curve, generated through separate system-level simulations. In a second scenario, the final upgrade is cell sectorization, going from 3 to $6 \mathrm{sec}-$ tors. In both cases a variant that includes the deployment of pico sites is also considered. Pico sites are especially useful in small spatial areas with high traffic densities. For this reason, when deploying pico sites, their location is based on a function that combines traffic density and areas suffering low user satisfaction rate. When considering a hybrid evolution path with new pico sites and macro upgrades, a fixed number of pico sites are first deployed, followed by any additional macro upgrades necessary to meet the KPI are carried out. With regard to the energy consumption of pico sites, energy models give an approximate ratio of 5:1 for the number of pico sites required to consume the same energy as a regular 3 sector single carrier macro site.

In all evolution cases, since the assumption is that all sites have the same type of equipment, network evolution always results in increased network energy consumption. By comparing the extent to which the energy consumption increases for the different scenarios, it becomes clear that some paths result in lower consumption. In order to put the capacity and data carrying capability of a network in contrast with the energy consumption, the term 'energy-efficiency' is defined. For a given network under full load, this term gives the energy cost for transferring a unit volume of traffic.

The purpose of a first HSPA evolution scenario is to determine the most energy efficient network evolution path between macro only upgrades, and a joint macro and (out-of-band) pico solution. In addition, the impact of different pico deployment densities is also investigated, with three categories being considered. In the network area, pico sites are deployed at the rate of 0 (macro only), 4, 8, or 12 sites per year.
Simulation results show that a macro only upgrade path leads to the greatest increase in energy consumption when compared to paths that include the deployment of pico sites. In the case when macro only upgrades are allowed, through additional carriers and MIMO, this results in a consumption increase of around $75 \%$. The cases of deploying 8, or 12 pico sites per year, gave comparable energy results, with the increase in consumption limited to $30 \%$. This mainly comes from the fact that deploying pico sites reduces the number of macro upgrades required (Figure 4), altogether removing the need for MIMO upgrades, the most energy expensive upgrade. When a large number of pico sites are deployed, use of the third carrier is not necessary either, leaving the network with possibilities of additional capacity upgrades. The heavy impact of MIMO on the energy consumption comes from the fact that a second, in the case of $2 \times 2 \mathrm{MIMO}$, parallel RF module is required.

A second network evolution case study is carried out on a 2 carrier HSPA layer, which in 2011 is complemented by LTE, deployed at all existing macro sites. Energy efficiency of the network is investigated on both the HSPA and LTE layers, allowing for 6-sector upgrades and/or the deployment of pico sites. Network evolution simulations are carried over a period of 9 years, following a traffic prediction growth model. The reference model portrays an average yearly traffic growth of $70 \%$, which is considered to be on the more aggressive side, while the second model considers an average yearly growth of $55 \%$, leading to an overall growth over the nine-year period of $\times 75$ and $\times 30$, respectively. Traffic is then split between the two layers (Figure 5), with LTE expected to surpass HSPA in terms of carried traffic around the year 2015-2016. The latest traffic predictions by CISCO support the aggressive traffic model, with 


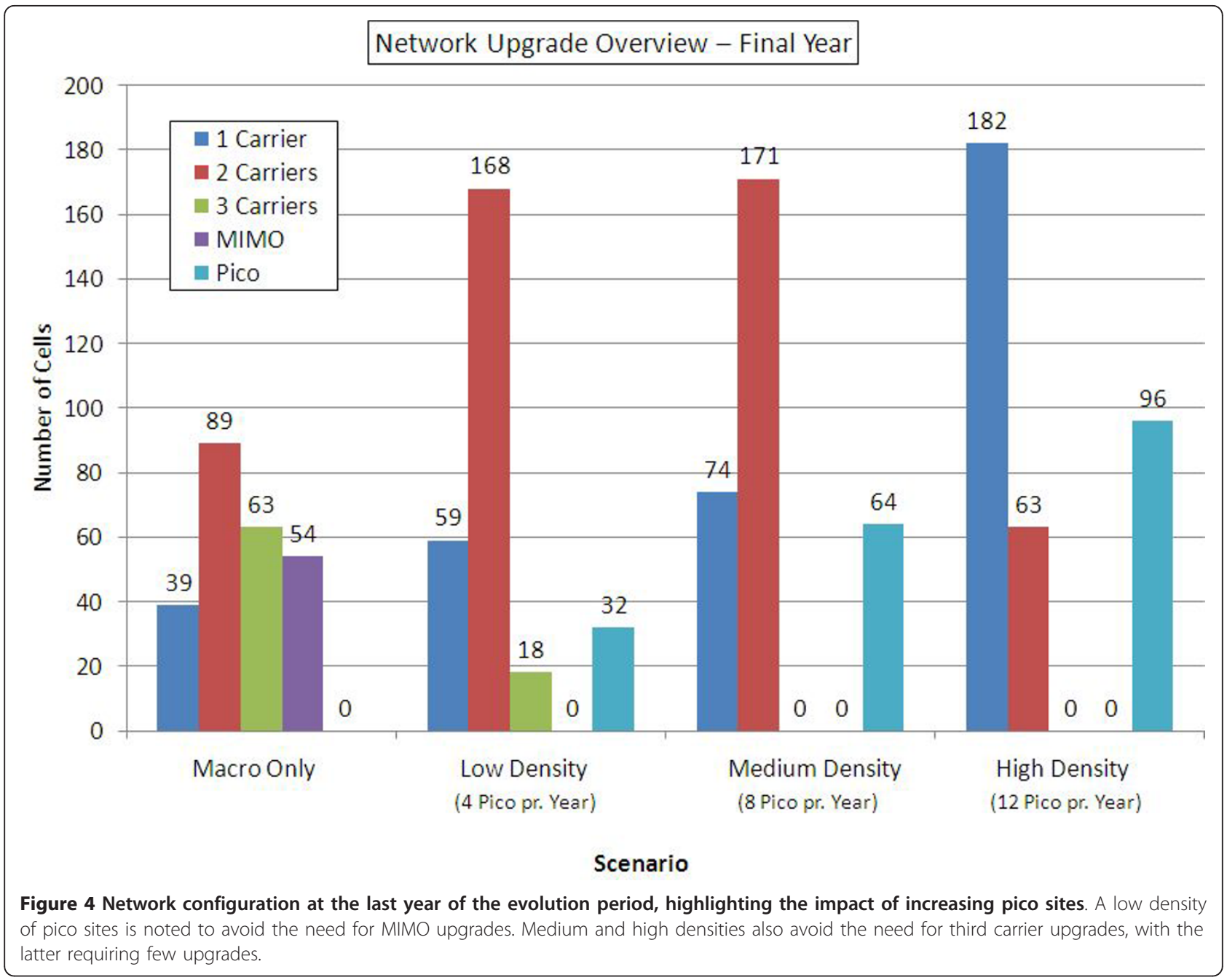

predictions foreseeing a growth by a factor of 26 between 2010 and 2015 [16].

In the case of an aggressive traffic growth, results show that a hybrid solution is the most energy-efficient, on both layers, with pico sites playing the role of offloading some of the macro sites in areas of high traffic density. By considering the rollout of LTE, and the upgrade of both HSPA and LTE layers, this leads to an increase in the energy consumption of the network, over a 9 -year period, by $200 \%$. In the case of a slower traffic growth, a pico only solution is sufficient and hence more energy-efficient over upgrading macro sites, with the increase in energy consumption limited at 132\% [17].

When upgrading the network for an aggressive traffic growth with a pico only solution, it is noted that a large number of pico sites are required to reach the required performance, making the solution unpractical. One of the key issues with small cells is how to provide the backhaul communication. While this depends on the country and infrastructure that the operator has, a simple cost analysis has been carried out for the HSPA network evolution for different pico densities. Cost data related to the equipment, installation, backhaul, and running costs for different upgrades are chosen based on discussions between the equipment vendor and operator. The selected backhaul assumption reflects the situation of most MNOs that do not own a fixed network and are required to lease capacity from other operators. Results show that the operational costs of a network increase dramatically as the number of deployed pico sites increase. On the other hand the macro layer upgrades require a higher initial capital expenditure, but have lower annual running costs [15].

\section{Replacing the old with the new}

In all energy-related studies it is assumed that all sites are the same, having the same setup and type of equipment. While this can be used to give an indication of the energy consumption of a network and the possible 


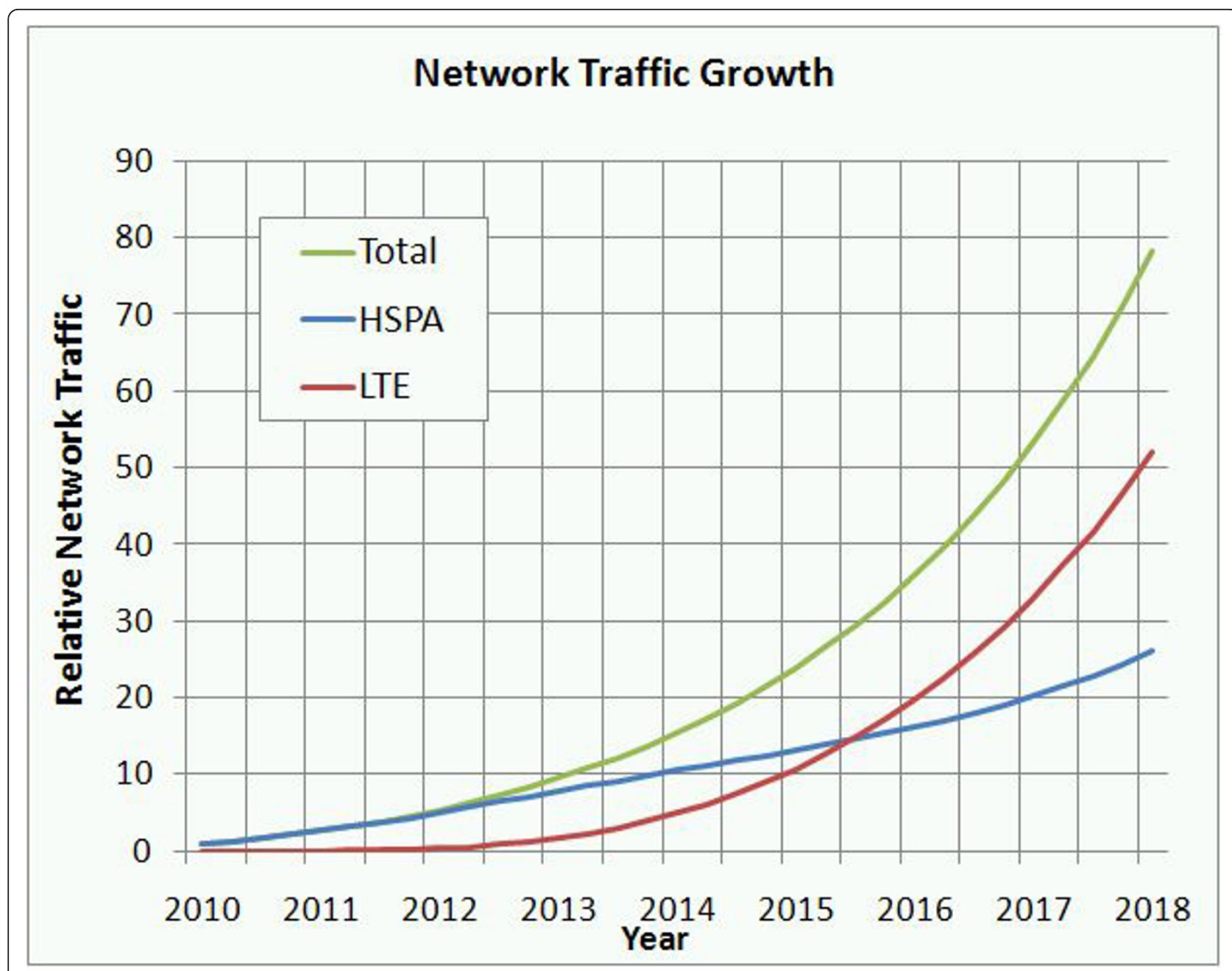

Figure 5 Traffic growth prediction model for the aggressive case (average of $70 \%$ annually), split between HSPA and LTE network layers. To note how the total growth between 2010 and 2015 matches that predicted by CISCO of $\times 26$.

gains of some features, this does not represent a realistic network energy consumption evolution trend. In a real network, MNOs manage different network layers, the sites of which have been deployed over a number of years. Some of the equipment at these sites has been there since the first rollout wave, whereas others would have been deployed for capacity or coverage upgrades at a later stage.

Over the last decade, a number of technological advancements have brought considerable equipment improvements, also in terms of energy consumption. Figure 6 gives an overview of how the energy consumption of base station equipment, setup in the same configuration, has evolved. This shows how two adjacent sites might have the same configuration and capacity, but one might be more efficient than the other. Since the rollout of $3 \mathrm{G}$ networks in the year 2000, a particular equipment vendor has released at least three versions of base station equipment, with considerable improvements in energy-efficiency from one release to another. This improvement means that MNOs can reduce the energy consumption of their networks simply by replacing older equipment with modern energy-efficient versions, absorbing parts of the increase in consumption by network upgrades. It is however interesting to understand what happens to the overall energy consumption of the network, if old equipment is replaced throughout the evolution of the network. Is it possible for MNOs to actually meet their targets of reducing the energy consumption, while at the same time meeting growing traffic demands?

\subsection{Energy gains through equipment replacement}

Based on the previous network evolution case study, the possible gains from equipment replacement are investigated. In these studies, base station sites are assumed to 


\section{Improvements in Equipment Power Consumption}

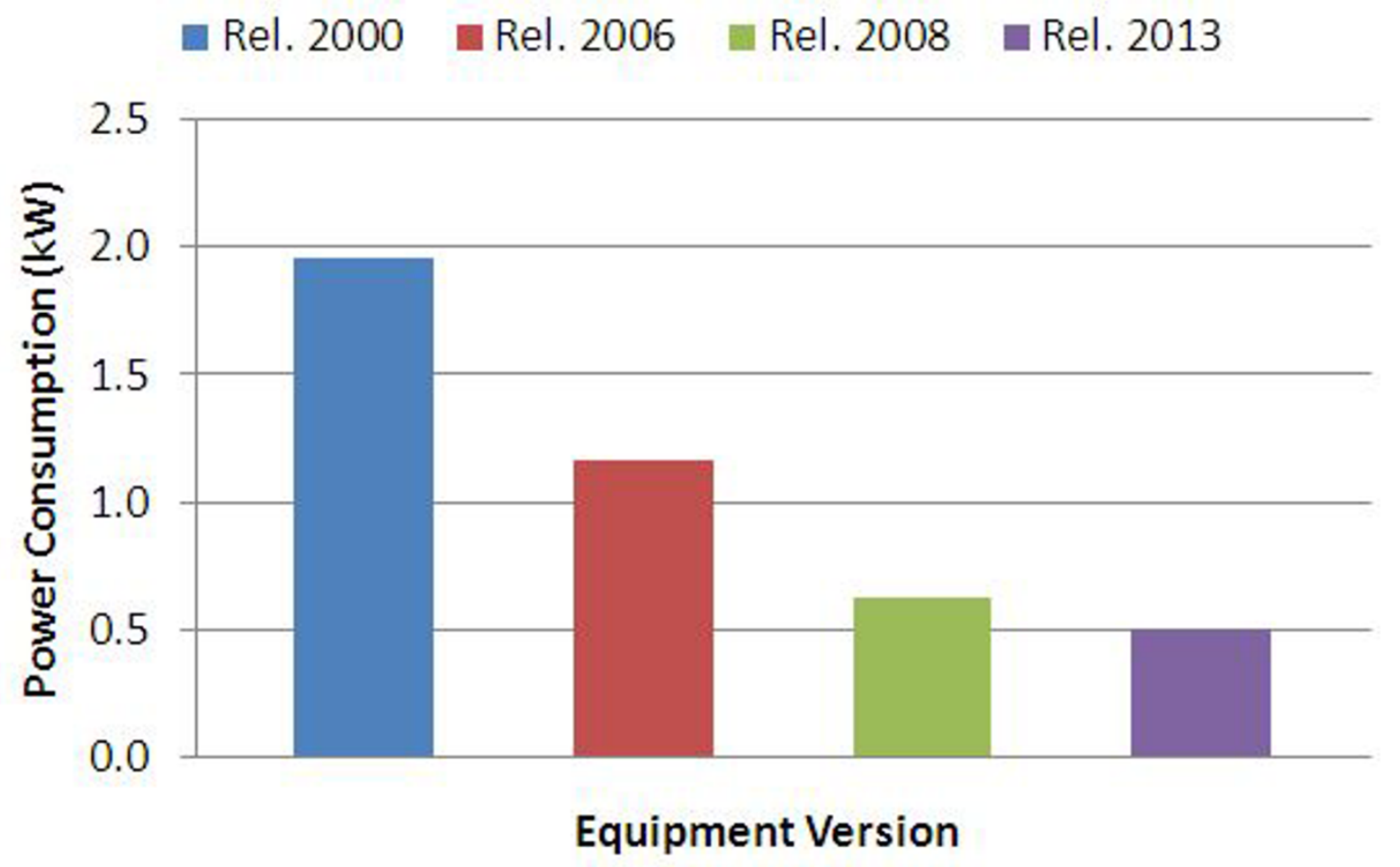

Figure 6 Site power consumption overview for different equipment versions, with same configuration ( 3 sector single carrier HSPA with no MIMO and RRH). The figure shows how over the last decade, advances in technology brought considerable improvements, which have slowed in more recent versions.

have the same type of equipment, specifically the version released in 2008, reference case, on which the energy models are based. Actual network data used to setup the simulation scenarios also reveals the type of base station equipment used at each site. For the considered case study, the network is composed of the first two generations of the equipment released in years 2000 and 2006, respectively.

The idea is to consider an equipment replacement strategy along the years, with the MNOs prioritizing the replacement of older and less efficient versions of the equipment. Network upgrades and the rollout of LTE are assumed to be carried out with the latest available equipment at the time. Besides replacing the equipment, this study also considers the option of upgrading sites with RRH units, an upgrade not considered in any of the previous cases. It is assumed that all sites equipped with the 2008 and 2013 versions of the equipment, have RRH units, thus reducing the feeder cable losses from 3 to $1 \mathrm{~dB}$.

In the previous study, the upgrade of an existing HSPA network, together with the rollout and upgrade of an LTE layer results in an increases energy consumption of $200 \%$. This considers an evolution period of 9 years, aggressive traffic growth prediction, and all sites having the same equipment version. The fact that an increase in carried traffic, by a factor of 75 , can be accommodated by increasing the energy consumption of the network by a factor of three represents a noticeable improvement in energy-efficiency, with $63 \%$ less energy required to transfer the same volume of data. Nonetheless, in comparison with the targets set by MNOs to reduce, and in some cases even half, their consumption, an increase by a factor of 3 does not help meeting these objectives.

In a first step, equipment replacement is carried out in a way to prioritize the gradual replacement of older equipment. The first version of the equipment is completely phased out within the first 3 years of the evolution period, whereas the second version (2006) is reduced to a $20 \%$ level, representing those sites that do not require any capacity upgrades over the evolution period (Figure 7, left). This also reflects other possible restrictions, primarily 


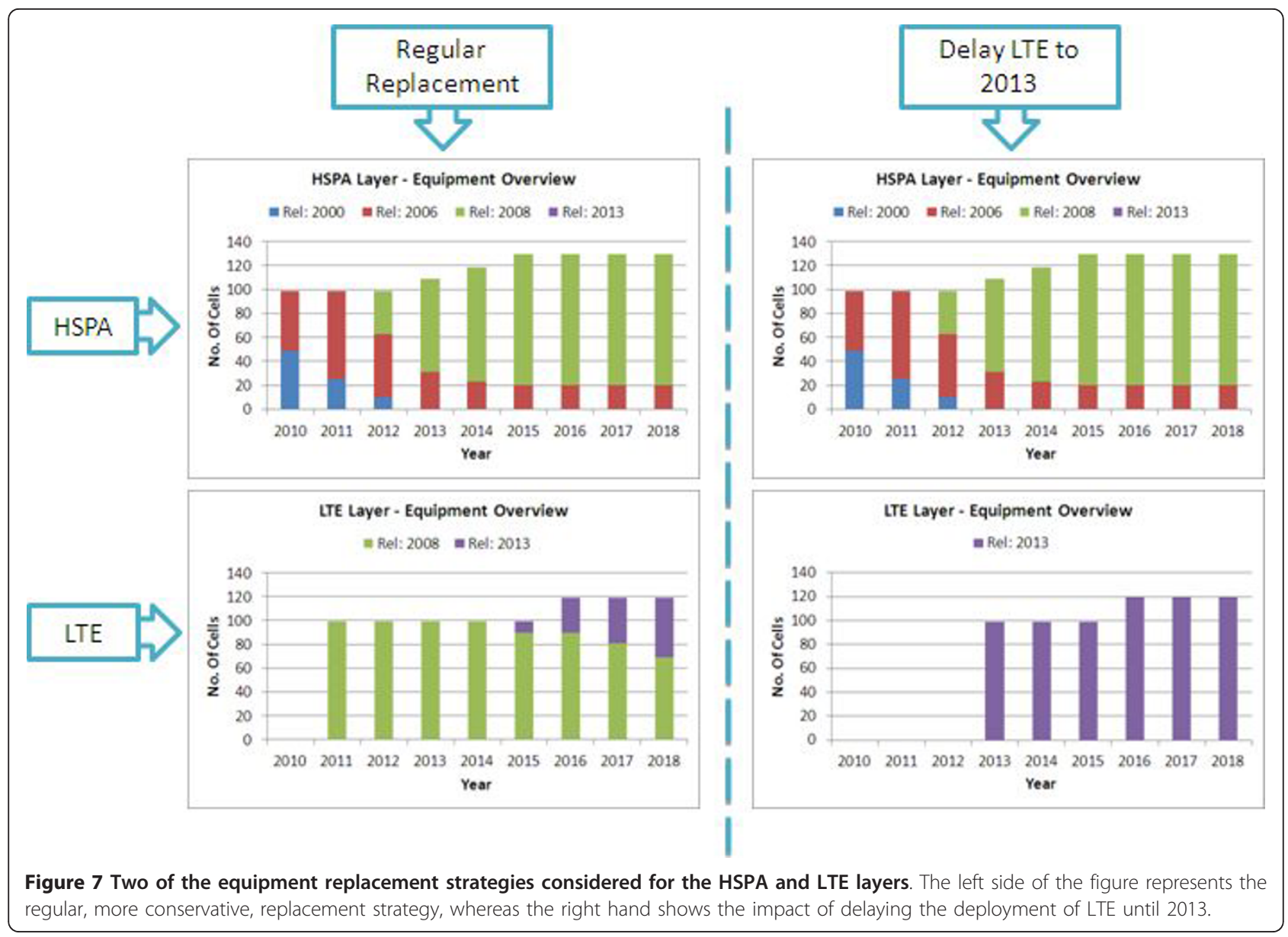

financial, since the operator is investing heavily in the LTE roll-out and capacity upgrades at other sites. Phasing out the year 2000 version of the equipment gives further savings, since it is assumed that no active cooling is required for the other versions of the equipment due to increased operational temperatures. While the extent of this varies from country to country, this assumption is based on the fact that in the case study area, temperatures are always within the operational temperature range of the equipment $\left(-35^{\circ} \mathrm{C}\right.$ to $\left.+55^{\circ} \mathrm{C}\right)$, meaning that passive cooling is sufficient for ensuring the safe operation of the equipment [18].

Energy consumption figures show, for the case with aggressive traffic growth, that the increase in energy consumption can, through equipment replacement, be limited from the original $200 \%$ to a mere $12 \%$. In contrast to previous single equipment assumptions, including older equipment increases the energy consumption during the first year even further, while modern equipment and RRH reduces it in the following years, effectively limiting the dynamic range between the first and last years. Since the same volume of traffic is assumed, these savings increase the energy efficiency of the network, reducing the amount of energy required for transferring the same unit volume of data.

Since the LTE layer is deployed very close to the release of a more efficient equipment version, a variation of this investigation looks at the impact of delaying the deployment of LTE until 2013 in order to exploit more efficient equipment (Figure 7, right). While it is assumed that this could trigger earlier HSPA upgrades, in the final years, the split between the two layers is kept unchanged as defined by the traffic growth model. This deployment delay brings an energy status-quo, meaning that the increase in carried traffic $(\times 75)$ is achieved at no extra energy cost. A further best case scenario is to change all equipment to the latest equipment when these are made available. This gives further savings, with an overall reduction in energy consumption of $20 \%$. This shows that a reduction in energy consumption while increasing network capacity is in fact possible. This amount of savings approach the targets set by some MNOs. These results and the equivalent for the slower traffic growth model are summarized in Table 2. 
Table 2 A summary of results comparing the evolution in energy consumption in an evolving HSPA and LTE mobile network, for two traffic growth models

\begin{tabular}{lll}
\hline & \multicolumn{2}{c}{ Change in energy consumption between $\mathbf{2 0 1 0}$ and $\mathbf{2 0 1 8}$} \\
\cline { 2 - 3 } & $\times \mathbf{7 5}$ Traffic growth (\%) & $\times \mathbf{3 0}$ Traffic growth (\%) \\
\hline Reference case & +200 & +132 \\
Regular replacement & +12 & -3 \\
Delay LTE to 2013 & 0 & -21 \\
Best case scenario & -20 & -38 \\
\hline
\end{tabular}

\section{Conclusions}

While the commitment of MNOs to reduce the amount of energy used for running their networks is undisputed, this article presents the key challenges and conflicts that exist. Since the need for network capacity upgrades is nevertheless crucial, leading to a further increase in energy consumption, operators have to achieve considerable energy savings through other methods. While no single solution is likely to put operators anywhere near their ambitious targets, realistic gains from a number of options have been investigated. These options include: energy-efficient network capacity evolution, energy saving through the replacement of old base station equipment, and the possible gains and limitations of an energy saving feature such as switching off base station sites. Results show that it is advisable for MNOs to upgrade the capacity of their networks through a solution that involves a combination of macro upgrades in conjunction with small cell deployment, providing savings of $25 \%$ when compared to a macro only solution. If during the evolution period old equipment is replaced, and upgraded with $\mathrm{RRH}$, this can result in a net energy consumption reduction ranging from 20 to $40 \%$. On a yearly basis, these results can be enhanced further through a range of energy saving features, exploiting network redundancies and traffic variations. Even though a feature such as switching off sites has potential for considerable savings (approximately 30\% daily), its implementation is currently impeded by technical limitations and risks of potential service deterioration.

This article brings together a number of practices which demonstrate that in a realistic network scenario, MNOs are actually capable of reducing the overall energy consumption of their networks. While other options are available (traffic steering, Wi-Fi offloading, etc.), the realization of these ambitious targets requires that equipment vendors keep improving the technology and efficiency of site equipment, and that MNOs give a higher priority to energy consumption when making network evolution decisions. In order to reach their $\mathrm{CO}_{2}$ emission reduction targets, it is likely that some MNOs might still need to invest in carbon-neutral energy to offset the difference.

\section{Abbreviations}

$\mathrm{CO}_{-2}$ : carbon dioxide; GSM: global system for mobile communications; HSPA: high speed packet access; KPI: key performance indicator; LTE: long term evolution; MIMO: multiple input multiple output; MNO: mobile network operator; OFDM: orthogonal frequency-division multiplexing; PA: power amplifier; PR: public relations; RF: radio frequency; $\mathrm{RRH}$ : remote radio head; RRM: radio resource management; SM: system module.

\section{Acknowledgements}

The authors would like to acknowledge that the work and results presented in this paper have been carried out as part of a doctoral research project at Aalborg University, Denmark, which is partly funded by Nokia Siemens Networks. In addition the authors have participated in and contributed to OPERA-Net, a Celtic-Initiative European consortium on the topic of energy saving in mobile networks. OPERA-Net has in December 2011 been extended by 3 more years under the new name OPERA-Net2.

\section{Author details}

${ }^{1}$ Radio Access Technology, Department of Electronic Systems, Aalborg University, Niels Jernes Vej 12 A6, 9220 Aalborg $\varnothing$, Denmark ${ }^{2}$ Nokia Siemens Networks, Aalborg, Denmark ${ }^{3}$ Nokia Siemens Networks, Kista, Sweden

\section{Competing interests}

The authors declare that they have no competing interests.

Received: 31 August 2011 Accepted: 6 February 2012

Published: 6 February 2012

\section{References}

1. Orange Labs, Towards a Greener World. (Orange Labs, 2010) http://www. orange.com/en_EN/responsibility/environment/. Accessed 30 Aug 2011

2. Operational Efficiency (Telenor 2010) http://www.telenor.com/en/corporateresponsibility/environment-and-climate/operational-efficiency/. Accessed 30 Aug 2011

3. Pressing Forward. Vodafone Group Plc Sustainability Report (March 2010)

4. F Richter, AJ Fehske, GP Fettweis, Energy efficient aspects of base station deployment strategies for cellular networks, Paper presented at the 70th IEEE Vehicular Technology Conference 2009, (Anchorage, USA, 2009), pp. 1-5

5. Y Chen, S Zhang, S Xu, G Ye Li, Fundamental trade-offs on green wireless networks. Huawei Technologies \& Georgia Institute of Technology. IEEE Commun Mag. 49(6), 30-37 (2011)

6. ABlresearch, Equipment and RF Power Device Analysis for Cellular and Mobile Wireless Infrastructure Markets. 4Q. (2010)

7. N Idir, Y Weens, J Franchaud, Skin effect and dielectric loss models of power cables. IEEE Trans Dielectrics Electric Insulat. 16(1), 147-154 (2009)

8. S Bhaumik, G Narlikar, S Chattopadhyay, S Kanugovi, Breathe to stay cool: adjusting cell sizes to reduce energy consumption, Alcatel-Lucent, Green Networking. (August 2010)

9. O Blume, H Eckhardt, S Klein, E Kuehn, WM Wajda, Energy savings in mobile networks based on adaptation to traffic statistics. Bell Labs Tech J. 15(2), 77-94 (2010). doi:10.1002/bltj.20442

10. G Micallef, P Mogensen, $\mathrm{HO}$ Scheck, Cell size breathing and possibilities to introduce cell sleep mode, Paper presented at the European Wireless 2010 Conference, (Lucca, Italy, 2010), pp. 111-115

11. A Brydon, M Heath, Will 3 G Networks Cope? Telecoms Market Research. (September 2009) 
12. J Halliday, C Arthur, Mobile operators signal end of flat-rate data tariffs as app use grows. The Guardian (2010). http://www.guardian.co.uk. Accessed 13 Dec 2011

13. Nokia Siemens Networks, LTE Performance for Initial Deployments. White Paper http://www.nokiasiemensnetworks.com (2009)

14. I Kovács, P Mogensen, B Christensen, R Jarvela, Mobile broadband traffic forecast modeling for network evolution studies, Paper presented at the 74th IEEE Vehicular Technology Conference 2011, San Francisco, pp. 1-5 (September 2011)

15. G Micallef, P Mogensen, HO Sheck, E Lang, Energy efficient evolution of mobile networks-macro-only upgrades vs. a joint-pico strategy, Paper presented at the 73rd IEEE Vehicular Technology Conference 2011, Budapest, pp. 1-5 (May 2011)

16. CISCO Visual Network Index: Global Mobile Data Traffic Forecast Update, 2010-2015, CISCO White Paper (February 2011)

17. G Micallef, P Mogensen, HO Sheck, Energy savings through site renewal in an HSPA/LTE network evolution scenario, Paper presented at the Wireless Innovation Forum, MVCE Green Radio Workshop, Brussels, Belgium, pp. 187-191 (2011)

18. Nokia Siemens Networks Flexi Multiradio BTS - All-purpose Flexi BTS featuring 3 technologies in 1, Nokia Siemens Networks http://www. nokiasiemensnetworks.com (2009). Accessed 13 Dec 2011

doi:10.1186/1687-1499-2012-34

Cite this article as: Micallef et al: Mobile operators have set ambitious targets-is it possible to boost network capacity while reducing its energy consumption? EURASIP Journal on Wireless Communications and Networking 2012 2012:34.

\section{Submit your manuscript to a SpringerOpen ${ }^{\circ}$ journal and benefit from:}

- Convenient online submission

- Rigorous peer review

- Immediate publication on acceptance

- Open access: articles freely available online

- High visibility within the field

- Retaining the copyright to your article

Submit your next manuscript at $\gg$ springeropen.com 